\title{
Synesthesia: from cross-modal to modality-free learning and knowledge.
}

by Roy Williams (researcher, academic), Dept. Mathematics, University of Portsmouth, PO1 3HF, UK: roytwilliams@gmail.com; Simone Gumtau (designer, academic), School of Art, Design \& Media, University of Portsmouth, Portsmouth, PO1 2DJ: simone.gumtau@port.ac.uk; Jenny Mackness (education consultant, teacher, researcher), c/o Roy Williams (see above): ienny.mackness@btopenworld.com.

ABSTRACT: In an integrated view of perception and action, learning involves all the senses, the interaction between them, and cross-modality rather than just multi-modality. In short: synesthetic enactive perception, which then forms the basis for more abstract, modality-free knowledge. This can underpin innovative learning design, and is explored in two case studies: children in Montessori preschools, and in the MEDIATE interactive space (for children on the autistic spectrum) in a 'whole body' engagement with the world. The challenge is to explore the rich opportunities offered by these modes of learning, and understand the transcriptions and transformations between them.

\section{INTRODUCTION}

Over the past 5 years, the authors have been researching how people learn, interact, and make sense of the world. We have drawn on insights from a range of theories and disciplines: affordances (from ecological psychology [1, 2, 3]), emergence (from complex adaptive systems [4, 5]), embodied learning (from phenomenology [6] and haptics [7]), communities and networks [8], and networked learning [9].

We see a thread running through all of this work, namely the idea that learning is more holistic and integrated across cognition, perception, context, affect and ontological development than previously acknowledged. Synesthetic ability and embodiment cut across all these areas, and enable us to reconfigure them into a more coherent framework.

We have been inspired by several authors' work on synaesthesia; particularly by the way Ramachandran extrapolates from involuntary synaesthesia (for instance, seeing numbers in colour), which we will refer to as synesthetic perception, to a much broader notion of synesthetic ability [10] (see below). Involuntary synesthetic perception clearly enriches many synesthetes' lives, and often inspires their imaginations in the creative arts $[11,12,13]$. However, it is the much broader, and somewhat different notion of synesthetic ability (or emergent cross-modal abstraction) that we are concerned with here. It is this broader notion that we will explore to see whether it can thrown light on our case studies of learning (see below).

\section{SYNESTHESIA}

Synesthesia was once seen as not much more than the product of some people's quirky imaginations. That has changed completely. Ramachandran shows why it is now scientifically recognised as an involuntary, fixed, and genuine sensory mode of perception, based on anomalous cross-wiring or cross-talk between different senses, and between different sensory areas of the brain. We will refer to this as (involuntary) synesthetic perception.

Ramachandran works on the hypothesis that synesthetic perception is based on individual genetic variation, and that 
broader synesthetic ability (see below) is based on the more general evolutionary development of particular areas of the human brain [14].

He distinguishes between two forms of synesthetic perception, depending on whether the gene/s are expressed lower down or higher up in the brain, resulting in synaesthesia between senses (e.g. colour, and the shape of numbers), in the Fusiform Gyrus, or between senses and concepts (e.g. colour and the concept of number, or "ordinality"), in the Angular Gyrus, respectively. These variants of synesthetic perception are both involuntary, and although they can be temporarily extended by hallucinogenic drugs, they are fixed, and cannot be learnt, or varied [15].

\section{Synesthetic Ability}

Ramachandran demonstrates, using Köhler's bouba/kiki experiment [16], that we all have some involuntary (e.g. graphic object-to-sound) synesthetic perception. What is important for this paper is the more generalised notion of synesthetic ability, which Ramachandran says is an extrapolation of these inbuilt biases that we all have, and is a characteristic of our evolutionary development. The bouba/kiki experiment shows that there is

a pre-existing translation between the visual appearance of the object ... and the auditory representation in the auditory cortex. In other words there's already a synesthetic cross-modal abstraction going on (+) ... Now admittedly this is a very small bias, but that's all you need in evolution to get it started and then you can start embellishing it [17].

He goes on to show that there are also pre-existing, built-in, cross-activation biases which map some shapes and sounds onto the motor maps in the Brocas area, and which similarly map movements between the hand area and the mouth area in the brain (first described by Darwin).

Such generalised cross-modal mapping is widespread, and is essential for the perception of all objects and movement in space, through perceptive-action [18] or enactive perception [19]; for language [20]; and for the social basis of learning [21, 22]. Merleau-Ponty argued that "synesthetic perception is the rule, and we are unaware of it only because scientific knowledge shifts the centre of gravity of experience" [23].

Ramachandran makes the case for a more flexible, emergent, complex, and creative synesthetic ability to identify and to create new patterns, which we all have (over and above the involuntary and fixed associations of synesthetic perception) and which is

not just a quirk in some people's brains. ... if you compare us [with animals ...] there is a progressive enlargement of the ... angular gyrus, ... strategically located at the crossroads between the parietal lobe (touch and proprioception), the temporal lobe (hearing), and the occipital lobe (vision); so it is strategically placed to allow a convergence of different sense modalities to create modality-free representations of things around you.

Once you develop this ability to engage in cross-modal abstraction, that structure in turn becomes an exaptation for other types of abstraction that [we] excel in, be it metaphor or any other type [24].

In other words, crucial parts of the brain which are involved in cross-mapping a range of sensory inputs give us our unique ability not only to perceive patterns (over and above associations) across the senses, but to use this ability and 
these basic synesthetic perceptual patterns to establish modality-free abstractions (metaphor, language, mathematics, and abstract and creative thought), or patterns of patterns. This (meta-) synesthetic ability also enables us to integrate our responses to the environment across these senses, using more complex synesthetic modes of communication such as speech and other media.

\section{Embodied cognition and enactive perception}

Many scientists and philosophers no longer consider mind and body to be separate from each other or from the environment, but rather as mutually interactive and adaptive. This applies across the 17 or so senses that Paterson identifies [25] rather than the 'five' discrete sense that we are still taught at school.

Traditional studies of cognition as computation have given ground to studies of embodied cognition, including ways in which the environment enables cognition, and the role of sensorimotor skills in cognitive development [26]. Most striking is the research by Cecilia Laschi [27], whose 'morphological computing' approach to the design of robots, assumes that intelligence resides not only in a central computing 'brain', but also in the 'body'.

Unlike many robots that have a hard humanoid shape, her robots have soft rubbery bodies which are able to move elegantly in water and respond flexibly to the environment, reminiscent of an octopus (Figure 1).

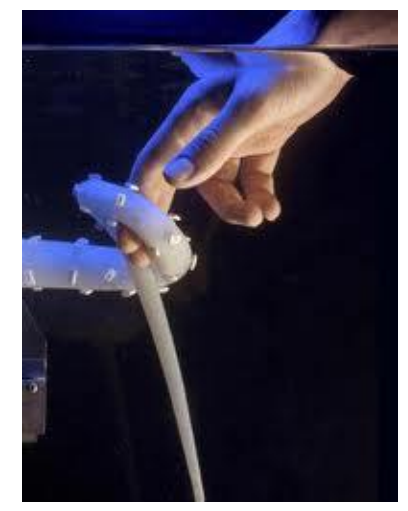

Figure 1: 'Octobot'

For Laschi, the octopus's intelligence is in its body and its interaction with the environment. As Mullins explains: "With morphological computing, it's not just the shape and substance of a body that's important, it's also the interaction with its environment that is crucial" [28] .

\section{CASE STUDIES}

Two case studies, Montessori and MEDIATE, explore the way these deas are applied in practice. In both cases the design includes, implicitly, substantial aspects of what (some years later) struck us as synesthetic ability.

\section{Montessori}

Introduction: The Montessori classroom was created in the early $20^{\text {th }}$ century for young children to harness their enthusiasm to explore the environment and interact with it. Working firstly with young children, with disabilities, Montessori responded to their need to interact physically with the world: embodied learning, using whatever facilities they had, with minimal instruction. 
The children explore patterns of physical coordination, then of material shape and form, which progress to more abstract patterns. There is no traditional 'instruction'. This grounds intellectual development in direct experience. She almost seems to have second-guessed Gibson's work on direct and enactive perception and meaning [29].

What is most radical is the lack of instruction or 'linguistic scaffolding': inviting the child, instead, to explore the senses directly: "The essential thing is for the task to arouse such an interest that it engages the child's whole personality" [30]. This anticipates Gibson's theory of affordances [31], which are neither 'in' the child nor 'in' the environment, but are rather the product of the interaction between of the two [32]. Costall points out that Gibson "appeals, in effect, to a [level of] a-linguistic and ultimately a-cultural ... human experience. ... Learning does not necessarily have to pass through the filters of linguistic coercion ... or explicit instruction" [33].

Examples: Substantial parts of Montessori mathematics, like the Trinomial Cube [a 3D rendition of the trinomial theorem, $(a+b+c)^{3}$ - Figure 2] are explored and mastered without any mathematical instruction, just a demonstration of the physical patterns, and an invitation to explore. The materials - literally, the blocks of wood function as a material, synesthetic or cross-modal scaffolding for the complex patterns of the theorem.

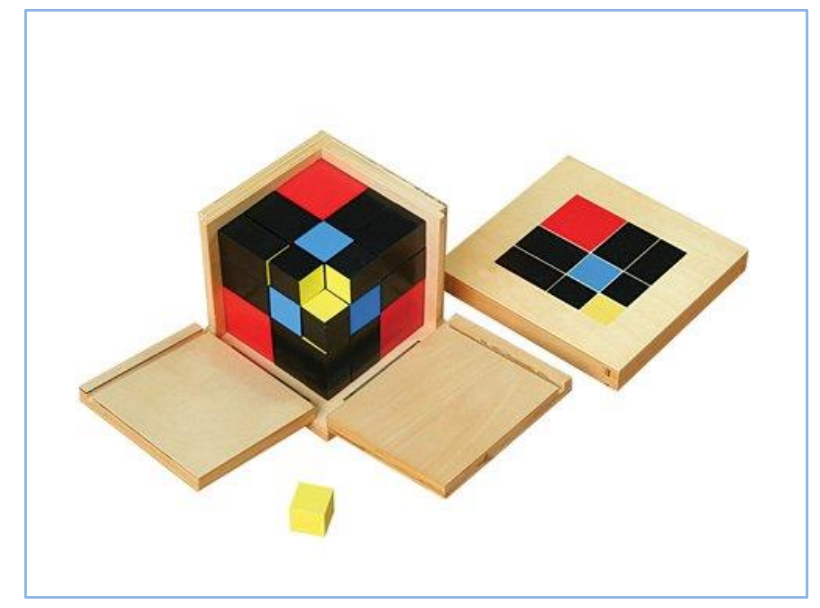

Figure 2: The Trinomial Cube

Comment: Applying synesthetic ability to the Montessori environment raises interesting issues. Kindergarten pupils are invited to interact with materials, most often in silence. They are not given complicated instructions, but rather invited to explore particular 'sensorial materials' and, effectively, their cross-modal affordances. The patterns they explore in the Montessori classroom range from gross motor coordination (scrubbing a table) to (literally) expanding (the patterns of) algebraic equations, in physical form.

The materials are carefully graded from synesthetically rich, 3D, haptic- and colour-intensive materials to more abstract patterns. These materials are, precisely, "strategically placed to allow a convergence of different sense modalities to create [an incremental series of abstractions, of increasingly] modality-free representations" - to paraphrase Ramachandran's definition of extended synesthetic ability [34]. For instance, principles of number, like base 10 are rendered in materials starting in 3D \& coloured, to 3D plain, to 2D coloured and/or plain, to 1D, to text, to numbers, and later to mathematical script, with built in recursion; the older child expanding the expression $(a+b+c)^{3}$ in algebra some years later often spontaneously walks 'back' to the trinomial cube, to its rich synesthetic patterns, to get a 'feel' for the theorem, and for the way 'unpacking' the cube 'expands' the pattern of $(a+b+c)^{3}$. 
This step-wise progression from rich, cross-modal multi-sensorial experience, to patterns, patterns of patterns and then modality-free patterns exploits synesthetic ability to the full.

\section{MEDIATE}

Introduction: MEDIATE (Multisensory Environment Design for and Interface between Autistic and Typical Expressiveness) was a multisensory environment designed for so called low-functioning children on the autistic spectrum with little or no verbal skills. It was a collaboration of five teams across Europe, involving designers, programmers, and psychologists. There was a plethora of theoretical perspectives and drivers [35]. Psychologically, it focused on developing agency, in an environment at once rich with sensory offerings, and devoid of any social context or symbolic content. The environment (Figure 3) was not designed to be therapeutic or to deliver a specific learning schedule, but to engage children with autism to feel in control of their space, enable them to play, and to explore novel behaviour and expressiveness. This was at all times driven by the needs of the child in dialogue with the environment. The achievement of dialogue with Autistic Spectrum children is a substantial achievement in its own right, and provides a substantial pedagogic and social advances, whether it achieves additional therapeutic goals or not.

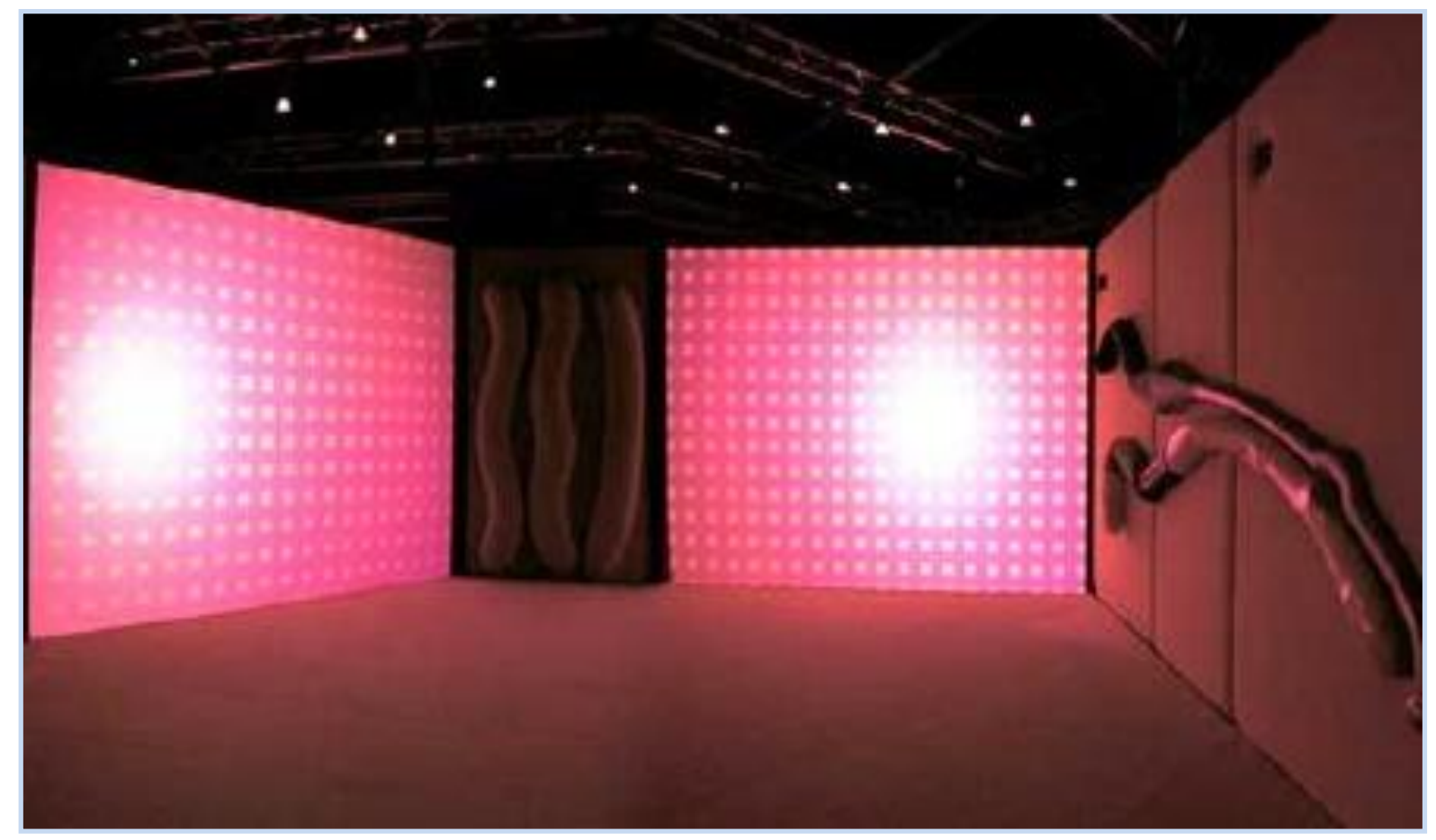

Figure 3: The MEDIATE Environment

It was also desirable that the parent or carer of the child could observe behaviour and expressions, learn about the child's sensory preferences, and perhaps have access to a world that is normally difficult for 'neuro-typical' people (i.e. without autism) to understand. Every individual with autism has their own unique idiosyncrasies, so it could be of great value for people close to a particular child to have a space to observe this sensory expression and communication.

Because the interactive agenda and dialogue was driven by the child at all times, the environment had to be absolutely accessible, without instruction or any social or cultural knowledge. As the premise was to allow novel and 
creative behaviour and expression to emerge, the interfaces and interaction had to move beyond button pressing and predetermined interaction sequences.

The solution was to have a fairly large, almost round space, which did not contain too many elements to fixate upon, but enough to experience and explore further. In terms of look and feel, the environment incorporated visual, audio and tactile interfaces, and integrated a range of organic and haptically interesting materials and shapes, and space to move and run around freely.

The system was intended to be intelligent and adaptive; pattern detection software was adapted to allow the system to build up unique, individual sensory profiles and to identify novel, idiosyncratic behaviour. The so-called 'brain' produced varied responses based on whether the child was inert, fixating and repetitive, or novel and explorative. This pattern detection was useful as it was un-biased by human observers, but the step from pattern detection to meaning making is still a substantial one, and realistically requires human monitoring.

The challenge was to implement rules within the system that allowed as much self-organization and adaptability as possible. If the child displayed novel, exploratory behaviour, the system increased the complexity of the interaction with the first entry into the environment being like walking into a sensory feedback loop, an amplification or extension of one's body. The interactive floor, for example, would produce footstep sounds, akin to walking on crunchy leaves, directly corresponding to the weight and gait of the person. As this continued, the nature of the sound changed slightly, into more complex, cross-modal responses - first a pitched crunch, later a singing voice.

Similarly, the 'Tunefork' (on the right-hand wall in Figure 3) would first amplify the sound the hand made on different textures (bark, cork etc), directly relevant to the material and the hand movement. This sound would then morph into more abstract sounds and cross-modal responses; e.g. in a later interaction stage, it was possible to tap the TuneFork to change the colour of the screen. This cross-modal aspect was important in the design process.

Designing a holistic sensory experience in an artificially controlled, technology-mediated environment presents challenges, as the experience has to be reduced into sensory channels to create interfaces with sensors and outputs. Content that must be intuitively understood has to be specifically designed and mapped across sensory modalities. For example, the concept of verticality is generally accepted as a so-called image schemata, which can be realised across different sensory modalities. When the child raises her arm vertically in space, it would make sense to map this gesture to a response on the screen - of a mirror silhouette raising an arm, but the environment might also respond arbitrarily and cross-modally, by 'raising' the volume or pitch of the sound - 'vertically' as it were. Cross-modal mappings have to be specifically designed in an artificial environment but this can allow the participant to discover or even create new mappings, a kind of embodied, synesthetic orchestration that would be engaging for the participant.

Examples: Many children used the MEDIATE space. These include two children we will call Mr Tunefork, and Mr Purple. Mr Tunefork is a 5 year old male without a diagnosis of Autism. His visit was remarkable in that without any musical training he commandeered the sounds produced by the Tunefork - a rather unusual 'instrument' in the traditional sense - to a high level of structured rhythm and pace. He moved up and down the bas-relief branch design (on the right wall in Figure 3), seemingly randomly touching and playing with the textures first, but then gradually composing a fairly complex sound-piece. 
Mr Purple is a 9 year old male with a diagnosis of Asperger's Syndrome, who had experienced the environment at several stages. At one point the interaction level had moved to a greater complexity and thereby cross-modality, and he was able to change the colour of the screens by tapping on the TuneFork. He proceeded to repeatedly select a purple hue - it might have gone unnoticed, but his mother observed it, and felt that this might be an important form of sensory expression. The family proceeded to paint his bedroom in this purple hue, and consequently experienced a much calmer child, able to sleep through the night for the first time in many years.

Comment: The children were nearly all on the Autistic Spectrum, and would be expected to experience strange, dark spaces as disorienting, if not frightening. None of them did. On the contrary, they intuitively found the space to be welcoming, inviting, and safe for exploration and expression - quite dramatically so. The choice of the pink colour, the soft, responsive, and varied surfaces - from underfoot to the walls; the soft lighting, and the soft or gently-inviting-andchallenging 'mind', all contributed to this.

Moreover, it was not a 'thing' to interact with, but on the contrary, a place to enter into, quite literally - in a deeply embodied (and possibly even a regressive) sense - that was soft, welcoming, and pneumatically inviting and responsive - for the feet, the hands, the face, and even the whole body, particularly in one case.

Within this space they found comfort at a rich, encompassing, and literally 'deeply synesthetic' and unmediated level what could perhaps be called Gibson's dream scenario for 'direct enactive perception'.

\section{DISCUSSION}

Synesthesic ability is not just an interesting curiosity; it provides us with the neurological basis for enactive, embodied, cross-modal learning and behaviour [36]. The case studies demonstrate long established, as well as recent, innovative, examples of the incorporation of synesthesic ability in learning design, in the Montessori classroom and in MEDIATE.

The Montessori pre-school shows how synesthesic ability can be rendered, 'embodied' and curated in learning materials. The child is provided with learning tasks which are incrementally more complicated, in small steps, in the materials, but also in small, incrementally more sophisticated abstractions - from the richest synesthetic crossmodality to the most abstract, modality-free texts. It's almost as if Montessori set out to enlist the Angular Gyrus as a proxy teaching assistant.

Clearly such rich integration of cross-modality is more applicable to some kinds of learners and stages of development (such as early childhood development) than others. But the value lies only partly in direct application. It also lies in a more general, creative awareness of the synesthetic, cross-modal foundations of learning, in the literal and metaphoric sense.

For instance, one of the authors had to teach 10 -year-old children who had little background or basic competence in mathematics to count in different number bases. The children were skipping competitively in the playground at the time, so the author got them to skip in the classroom for two days, counting in different number bases. They soon had no problem counting in any number base you cared to throw at them, even while they were skipping. 
And similarly, Quinn's research into young people on the margins of education [37] describes learners who floundered in the sensorially and synesthetically impoverished, abstract setting of text-based schooling, which assumes that the transition from rich embodied cross-modal experience to written text is natural, comfortable and selfevident for everyone. Quinn's learners, too, thrived as soon as they were put (back) into rich synesthetic settings: in their case, in a farm-based school. They seemed to have been yearning for the 'reinstatement of all their other (16 or so) senses' [38]. They were potentially quite capable of engaging with written text, given the right guidance and time, but they experienced the 'cold turkey' approach of schooling and the 'shock of the text' as intolerable and disorientating.

MEDIATE, on the other hand, shows how it is possible to embody synesthetic ability and enactive meaning in an interactive space, which 'in-corporates' the learner in an immersive, simulated synesthetic presence, built around its own 'virtual angular gyrus', so to speak. This simulated presence is not 'external' to the learner; the learner literally enters into it, by going into a womb-like space, and interacting from the 'inside'.

\section{Comfort and Agency}

MEDIATE's rich, encompassing, and 'deeply synesthetic', unmediated zone provides a 'home' for the development of autonomous agency, and the expression of a creative, engaging, embodied self in 'dialogue' with an 'other'. Happé, a member of the MEDIATE team, formulated it like this:

Ordinary young children ... have the sense of agency [early on] ... in the social world. A very young infant from four months can smile at a parent, the parent smiles back, they immediately have a sense of acting on the world ... and that's long before they can control their motor movements and affect the world in that way ... Children with autism, partly because of their social problems, don't have such an experience of control over the world. ... the world for them is typically chaotic and unpredictable. So the point of MEDIATE ... is to give the child an experience of being utterly in control, where their world is suddenly no longer chaotic (personal communication, 2002).

The MEDIATE 'brain', or mind, satisfies some of the criteria for other (cognitive) minds (as in 'Theory of Mind' research), in which the participant has to best guess what is going on in the other 'mind' they are interacting with. In MEDIATE the dialogue was based on the contingency (or randomised variation) built into the programme - which had to be adaptive and finely tuned to achieve a 'Goldilocks' level of contingency (not too much, not too little, but 'just right'), and invite and guide the participants out of repetitive loops, and into creative, expressive space.

However, the achievement of a fundamental, intuitive level of comfort-in-a-strange-place introduces quite different dynamics to those involved in a 'cognitivist' Theory of Mind interaction. Experience in MEDIATE seems to overlap with what Turkle [39] describes as emotional engagement and projection, in which people not only develop affection for what we might call 'ET-bots', but they experience the ET-bots as reciprocating the emotional engagement, and 'loving' them back. We might call this Theory of Heart.

This raises a further issue: is MEDIATE just an example of Theory of Mind + Theory of Heart, or is there something more fundamental and integrative at stake, which we could call a 'Theory of Synesthetic Mind' - one which deliberately and sensitively accentuates cross-modality in its responses? We might learn something from this that 
would be applicable to 'neuro-typical' children too, just as Montessori's early work with disabled children provided the foundations for early learning for all children.

\section{CONCLUSION}

The two case studies share a radical notion of synesthesic ability, starting with cross-modality and extrapolating into the modality-free abstractions of language and knowledge - implicit in design, explicit in realisation. Both provide a starting point for engagement, which is embodied and synesthetic. In MEDIATE, embodiment is taken to a new level: of immersive embodiment of body/mind - so much so that we might need a new term ('embodiedmind'). In both cases the core engagement is embodied, material, direct and intuitive; in both cases the standard 'linguistic and cognitive scaffolding' is designed out - as far as possible, to enable what Gibson aspired to: direct enactive perception and meaning [40], exploiting layers of cross-modality to the full, in 'synesthetic scaffolding' and even 'synesthetic orchestration', leading to modality-free expression in abstract patterns.

Synesthesic ability can potentially enrich learning by providing affordances for perceiving and engaging intuitively with the world as well as the crucial capability to explore, express and engage in dialogue with the internally motivated self, building up comfort, agency and capability.

The two cases differ in the extent to which they engage with the transcription and abstraction of patterns beyond rich synesthetic engagement, although both are radical in their approaches and solutions. MEDIATE focuses on 'putting the child in charge' within a synesthetic experience inside a (virtually) simulated 'embodiedmind', which generates emergent sensory and cross-modal traces - potential transcriptions, 'texts' and even transformations that can be taken further, e.g. Mr Purple.

The Montessori example shares these concerns for the primacy of ontological development, agency, and self as a site for engagement and dialogue with the world. In the Montessori classroom, as children establish agency they move on to other modes of interaction and knowledge. Montessori materials are meant to be extended to engage the child in emergent stages of learning, building on a foundation of rich synesthetic cross-modality, and moving on to modalityfree abstraction.

The value of the framework of 'embodied synesthesic ability' lies in the fundamental nature of enactive perception and enactive meaning, and the role it can play in various forms - from the 'immersive' synesthesia of a MEDIATE-like environment to the very different Montessori environment in which engagement ranges from the rich synesthetic to the abstract (and back - in re-engagement and in recursion).

\section{Implications for future research}

Synesthesia foregrounds two modes of active engagement with the world: cross-modal, and modality-free. This is based on the progression and extrapolation from involuntary synesthesic perception across senses, to involuntary synesthesic perception across senses and concepts, to broader synesthesic ability, which identifies and creates completely new, modality-free abstraction. 
This raises interesting questions about how largely intuitive engagement, even in the algebra of the trinomial theorem, can provide rich, cross-modal forms of engagement for what is in principle modality-free knowledge (albeit in the more abstract forms of mathematics).

This is contrasted against the (Peircian) 'symbolic' engagement of the abstract, modality-free written text, which has its own power in its ability to articulate totally new knowledge, through the arbitrary-and-conventional nature of language and semiotics. It is also contrasted against what might be called the 'intuitive-aesthetic' engagement of art, which is paradoxically more abstract and more intuitive than the symbolic, for example and the work of artists like Brancusi (see Figure 4).

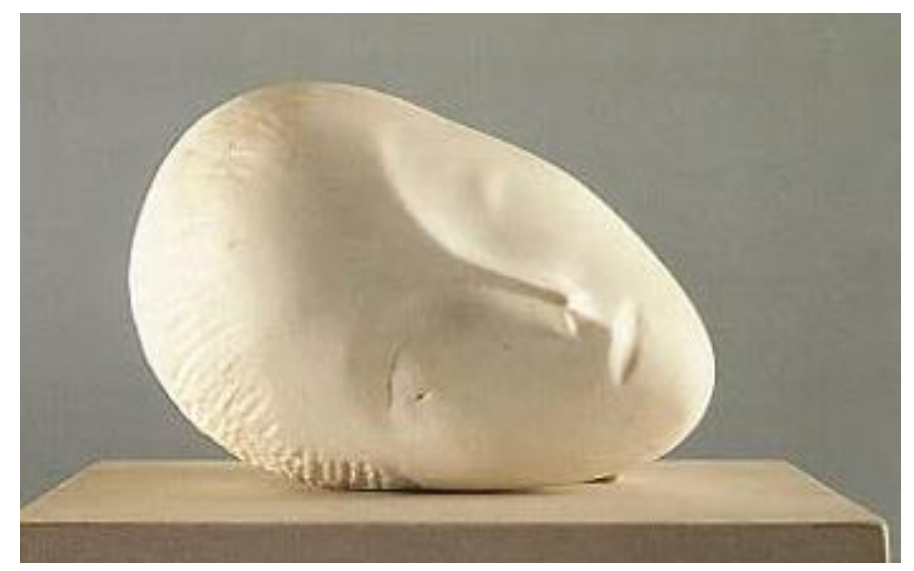

Figure 4. Brancusi's Head

This is in a sense a 'pure' or 'open' form. Your first response is intuitive, and an explanation might diminish your appreciation rather than enhance it, despite the rich intertextuality. Brancusi might almost have invented the term 'intuitive-aesthetic'. He writes that "there are idiots who define my work as abstract; yet what they call abstract is what is most realistic. What is real is not the appearance, but the idea, the essence of things." [41].

Pure mathematics can function in a similar mode, discovering mathematical patterns which capture relationships in the abstract which have their own elegance and beauty, e.g. Mandelbrot fractals, which often relate to phenomena that may only be scientifically described many years later [42].

What is important is to recognise that all three modes: intuitive, symbolic and intuitive-aesthetic provide rich, powerful modes of engagement, situated in, or derived from the rich spectrum of synesthesic ability that we have explored here, and that there is work to be done in leading learners from the one stage to another.

\section{REFERENCES}

1. J.J. Gibson, The Ecological Approach to Visual Perception (Boston: Houghton Mifflin, 1979).

2. A. Noë, Action in Perception (Cambridge, MA: MIT Press, 2005).

3. A. Costall \& O. Dreier, Doing Things with Thing (Aldershot, UK: Ashgate, 2006).

4. P. Cilliers, "Complexity, deconstruction and relativism," Theory, Culture and Society, 22(5), 255-267 (2005).

5. D.J. Snowden, \& M. Boone, "A leader's framework for decision making," Harvard Business Review (2007).

6. M. Merleau-Ponty, Phenomenology of Perception (London and New York: Routledge, 1962) p. 229. 
7. M.W. Paterson, The Senses of Touch: Haptics, Affects and Technologies (Oxford: Berg Publishers, 2007).

8. E. Wenger, Communities of practice. Meaning, learning and identity (USA: Cambridge University Press, 1998).

9. J. Dron \& T. Anderson, "Lost in social space: Information retrieval issues in Web 1.5" Journal of Digital Information, 10(2), (2009).

10. V.S. Ramachandran, The Emerging Mind: Purple Numbers and Sharp Cheese. Reith Lectures (2003). Retrieved 2012-07-16.

11. C. van Campen, The Hidden Sense: Synesthesia in Art and Science (Cambridge Mass: MIT Press, 2008).

12. R.E. Cytowic, Wednesday is Indigo Blue: Discovering the brain of synesthesia (Cambridge Mass: MIT Press, 2009).

13. J. Ward, The Frog Who Croaked Blue: Synesthesia and the mixing of the senses (London: Routledge, 2008).

14. Ramachandran [10].

15. Ramachandran [10].

16. W. Köhler, Gestalt Psychology (New York: Liveright Publishing Corporation,1947).

17. Ramachandran [10].

18. Gibson [1].

19. Noë [2].

20. M. Marshall, "Chimp prodigy shows signs of human-like intelligence," New Scientist, (2012).

21. A. Rogowska, "Categorization of synesthesia," Review of General Psychology 15(3), 213-227 (2011).

22. C. Heyes, "New thinking: the evolution of human cognition," Philosophical Transactions of the Royal Society B:

Biological, 367 (1599), 2091-2096 (2012).

23. Merleau-Ponty [6].

24. Ramachandran [10].

25. Paterson [7].

26. M. Cowart, Embodied Cognition (2005). Retrieved 2012-07-16.

27. C. Laschi, M. Cianchetti, B. Mazzolai, L. Margheri, M. Follador \& P. Dario, "Soft Robot Arm Inspired by the Octopus," Advanced Robotics 26 (7), 709-727 (2012).

28. J. Mullins, "Squidgybots," New Scientist, p.50 (November 2011).

29. Gibson [1].

30. M. Montessori, The Absorbent Mind (New York: Dell, 1967 edn., 1949) p. 206.

31. Gibson [1].

32. R.T. Williams, Affordances and the New Political Ecology. In M. Taylor \& P.M. Currie (eds): Terrorism and Affordance (London: Continuum, 2012) p. 93-120.

33. A. Costall, "Socializing affordances," Theory and Psychology, 5, 467-481, p.472 (1995).

34. Ramachandran [10].

35. S. Gumtau, P. Newland, C. Creed \& S. Kunath, MEDIATE. A Responsive Environment Designed for Children with Autism. Accessible Design in the Digital World Conference, Dundee, Scotland (2005).

36. Ramachandran [10].

37. J. Quinn, "Theorising Learning and Nature," Paper at The Future of Theory in Education, Stirling University, Scotland (2012).

38. Paterson [7].

39. S. Turkle, Alone Together (New York: Basic Books, 2011).

40. Ramachandran [10].

41. C. Brancusi, Caiete Silvane magazine. (2009). Retrieved 2008-11-01.

42. A. Osbaldestin, The unreasonable beauty of mathematics. Inaugural lecture, University of Portsmouth (2009). 